\title{
Research on reverse supply chain problem based on immune algorithm
}

\author{
Yunkai Zhong a, Yang Zhang ${ }^{b}$ \\ Wuhan University of Technology, Wuhan 430000, China. \\ a2653977145@qq.com, b1115169488@126.com
}

Keywords: Reverse Supply Chain, Immune Algorithm, Localtion Selection.

\begin{abstract}
Recycling is the key link in the reverse supply chain. Recycling center is a bridge between suppliers and customers. Recycling center's location often determines the recovery, distribution distance and distribution mode of logistics. In order to improve the efficiency and reduce the logistics supply costs, the immune algorithm applied to the recycling center relocation and verify the correctness with examples Achieve reverse recovery of products.
\end{abstract}

\section{Introduction}

Reverse supply chain refers to a series of activities that recover products from consumers and discard or recycle the recovered products. However, companies are forced to set up reverse supply chains under environmental or consumer pressure. The purpose is to dispose of recycled products or reuse. Research shows that effective reverse supply chain can enhance customer satisfaction, while increasing corporate profits. One of the returned goods handling, which is a part of reverse logistics has become a lot of enterprises to enhance the competitiveness. As long as companies carefully analyze their own reverse logistics chain, they may find there are many improvements.

The location of the recycling center often determines the courier logistics recovery, delivery distance and recovery, delivery methods, thus affecting the courier recovery, delivery system operational efficiency. Moreover, once the location of the recycling center has been identified, its location is difficult to change. Therefore, it has very important theoretical and practical significance to solve the establishment of courier logistics distribution and recycling center location. Recycling center location model is a non-concave and non-smooth nonlinear programming model with complex constraints, which belongs to NP-hard problem. According to the courier location information and courier traffic and other information, We bulit the immune algorithm. Determine the specific location of the recovery point, and recycling center which is responsible for the distribution point position and quantity.

\section{Establishment of Logistics Distribution Center Location Model}

In the logistics distribution center model to make the following assumptions:

The size of the distribution center can afford the needs of demand points.

Ignore the shipping costs from the factory to the distribution center.

A logistics demand point is supplied by only one distribution center

Based on the above assumptions, we establishe a logistics distribution center location model. The model solves two problems. One is to determine the location of the distribution center, the other is the distribution logistics point number. We need to find distribution centers from $n$ logistics demand points and deliver projects to each demand center. The objective function is to minimize the product of demand and distance from each distribution center to the demand point.

The objective function is as follows

$$
\min F=\sum_{i \in N} \sum_{j \in M_{i}} \omega_{i} d_{i j} X_{i j}
$$

Restrictions: 


$$
\left\{\begin{array}{c}
\sum_{j \in M_{i}} h_{i}=p \\
\sum_{j \in M_{i}} Z_{i j}=1 \quad, \quad i \in N \\
Z_{i j} \leq h_{i} \quad, \quad i \in N \quad, \quad j \in M_{i} \\
Z_{i j}, \mathrm{~h} \in\{0,1\} \quad, \quad i \in N \quad, \quad j \in M_{i} \\
d_{i j} \leq s
\end{array}\right.
$$

In the above formula, $N$ represents the set of all demand point. $M_{i}$ is the set of alternative distribution centers for which the demand point $i$ is less than $s ; M_{i} \subseteq N ; \omega_{i}$ represents the demand point demand; $d_{i j}$ represents the nearest distance from demand point $i$ to distribution center $j ; Z_{i j}$ is a $0-1$ variable. When it is 1 , it indicates that the demand of demand point $j$ is supplied by distribution center $j$, otherwise $Z_{i j}=0 ; h_{i j}$ is alse a $0-1$ variable. When it is 1 , it means that point $j$ is selected as the distribution center; $s$ is the distance between the new distribution center and the demand point served by it.

\section{Establishment and Solution of Immune Algorithm}

\subsection{Introduction of Immune algorithm}

Artificial immune system is an interesting bio-inspired intelligent approach that simulates the information processing procedures of biologic immune system [1,2]. From a computational perspective, the biological immune system with strong learning, recognition and memory capabilities. Among them, artificial immune algorithm is designed by mimicking the information processing procedures of artificial immune system (AIS), which has attracted much attention and been successfully applied in many research fields, such as data mining, computer security, optimization, and fault diagnosis [36,37]. Especially for tackling MOPs, artificial immune algorithm has demonstrated to be very competitive as experimentally studied in [3-4].

\subsection{Initial antibody population generated}

We used a simple coding approach to form an antibody of length $l$ ( $l$ is the number of distribution centers) for each site selection scheme. Each antibody representing the sequence of demand points selected for the distribution center.

\subsection{Solution diversity evaluation}

First, we use the affinity between the antibody and the antigen to indicate the degree of recognition . In this model, we devised an affinity function $V$.

$$
V=\frac{1}{\sum_{i \in N} \sum_{j \in M_{i}} \omega_{i} d_{i j} Z_{i j}-C \sum_{i \in N} \min \left\{\left(\sum_{j \in M_{i}} Z_{i j}\right)-1,0\right\}}
$$

$V$ is the reciprocal of $F$. The second of the denominators indicates the penalty for solutions that violate distance constraints. So the value of $C$ is relatively large.

Second, we use the antibody concentration $C_{v}$ to represent the proportion of similar antibodies in the population.

$$
C_{v}=\frac{1}{N} \sum_{j \in N} S_{v, s}
$$

$N$ represents the total number of antibodies; when $S_{v, s}$ is greater than $T, S_{v, s}$ is 1 , and vice versa; $T$ is a pre-set threshold.

Last, we introduce the expected probability of reproduction. In a population, the expected multiplicity of reproduction of each individual is determined by the affinity of antibody and antigen, $V$, and antibody concentration $C_{v}$. 


$$
P=\alpha \frac{V}{\sum V}+(1-\alpha) \frac{C_{v}}{\sum C_{v}}
$$

$\alpha$ is a constant. Through the above formula, we can analyze that the higher the fitness of individuals, the greater the probability of breeding.

\subsection{Immune operation}

First of all, we use the roulette method to select individuals. Then we make a single point crossover to the chosen individuals. Finally, the crossover results are mutated to increase diversity.

\subsection{Algorithm flowchart}

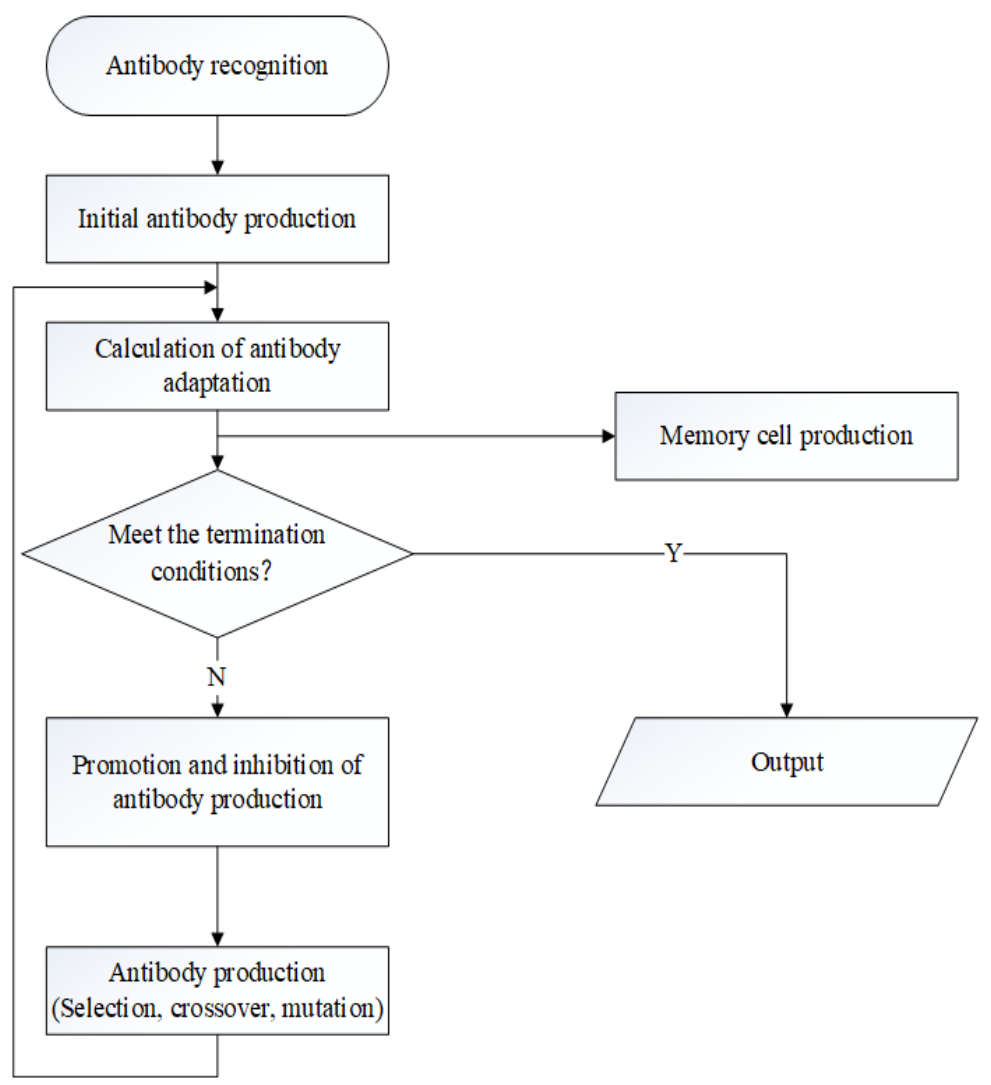

Fig 1Algorithm flowchart

\section{Literature References}

In order to verify the correctness and validity of the above algorithm, we use 79 logistics delivery points $Q_{1}, Q_{2}, \ldots, Q_{79}$, with the longitude of $\mathrm{x}_{1}, \mathrm{x}_{2}, \ldots, \mathrm{x}_{3}$, latitude of $\mathrm{y}_{1}, \mathrm{y}_{2}, \ldots, \mathrm{y}_{3}$, circulation capacity were $r_{1}, r_{2}, \ldots, r_{3}$, The specific data shown in the table below:

Table 1 The cities condition

\begin{tabular}{|c|c|c|c|}
\hline Supply station & longitude & latitude & Circulation \\
\hline$Q_{1}$ & 1304 & 2312 & 26 \\
\hline$Q_{2}$ & 3639 & 1315 & 12 \\
\hline$Q_{3}$ & 4177 & 2244 & 59 \\
\hline$\ldots \ldots$ & $\ldots \ldots$ & $\ldots \ldots$ & $\ldots \ldots$ \\
\hline$Q_{77}$ & 1807 & 2993 & 19 \\
\hline$Q_{78}$ & 4185 & 3258 & 48 \\
\hline$Q_{79}$ & 3023 & 1942 & 60 \\
\hline
\end{tabular}

Calculated using the above method, the termination of the algebra 100, the optimal number of individuals 34, selected 14 optimal distribution center is selected, the results as shown below. 


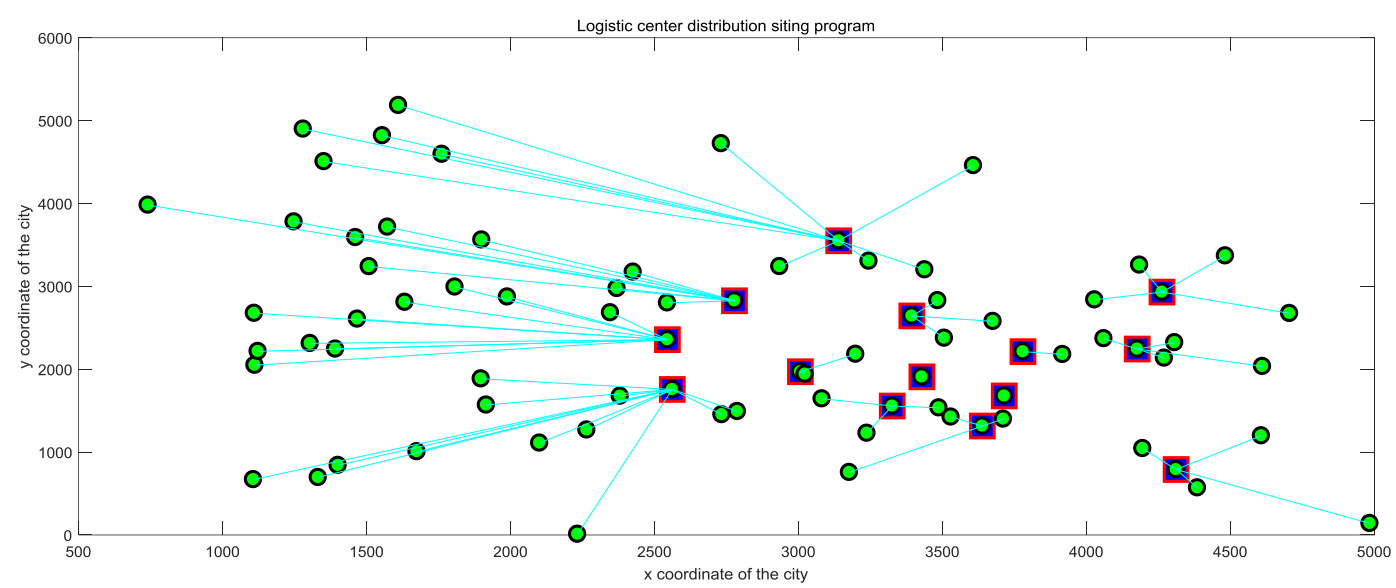

Fig. 2 Results Figure

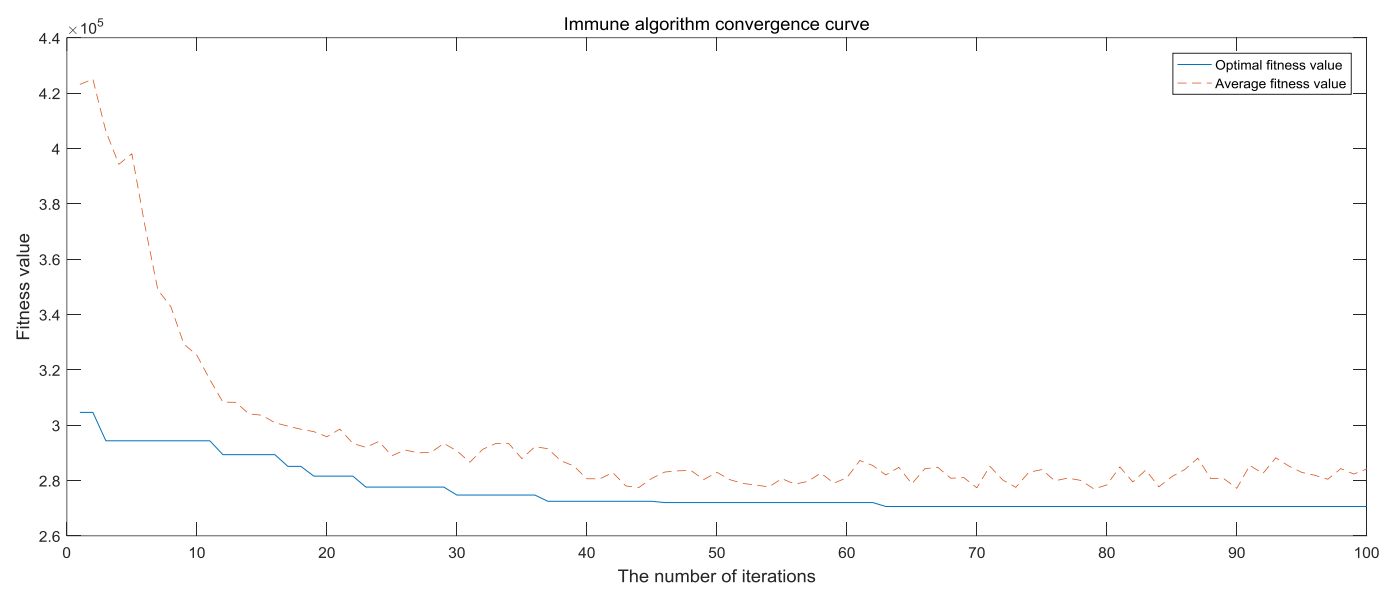

Fig. 3 Convergence Figure

Thus, using genetic algorithm to solve the problem of distribution center location, and achieved good results.

\section{Summary}

In this paper, examples are used to verify the correctness of the model and the effectiveness of the immune algorithm. The optimal solution to the problem is obtained, and the address and traffic of the distribution center with the lowest total cost are obtained, which effectively solves the problem of the location of the distribution center. The problem not only involves the location of the distribution center and the determination of the distribution plan. But also involves the cost of constructing distribution centers in different locations and the reasonable choice of distribution centers to minimize costs and other factors. It is a complicated system optimization problem. As a method of solving optimization problems, immune algorithm provides a brand new tool for the calculation of complex systems. It can solve the problems of delivery planning and optimal delivery plan at the same time, has good practical value and wide application prospect.

\section{References}

[1] L.N. De Castro, J. Timmis. An artificial immune network for multimodal function optimization. Proceedings of the 2002 Congress on Evolutionary Computation, 2002, pp. 699-704.

[2] L.N. De Castro, F.J. Von Zuben. Learning and optimization using the clonal selection principle. IEEE Trans. Evol. Comput. 6 (3) (2002) 239-251.

[3] M.G. Gong, L.C. Jiao, H.F. Du, L.F. Bo. Multi-objective immune algorithm with nondominated neighbor-based selection. Evol. Comput. 16 (2) (2008) 225-255. 
[4] R.H. Shang, L.C. Jiao, F. Liu, W.P. Ma. A novel immune clonal algorithm for MO problems. IEEE Trans. Evol. Comput. 16 (1) (2012) 35-50. 